\title{
Signaling unearned reinforcers removes the suppression produced by a zero correlation in an operant paradigm
}

\author{
LYNN J. HAMMOND and MICHAEL WEINBERG \\ Temple University, Philadelphia, Pennsylvania
}

\begin{abstract}
Rats in two separate experiments were trained on schedules of reinforcement that allowed the probability of reinforcement (a drop of water) given a leverpress in any second and the probability of water given no leverpress in any second to be manipulated independently of one another. After training on high-positive contingencies (no water for not leverpressing), rats were shifted to zero contingency (equal water for pressing or not pressing), and, as is usually found, leverpressing declined radically. Then each water reinforcer given for not leverpressing was signaled by a 5-sec flashing light and gradually, after many sessions, leverpressing returned to normal. This effect was seen for all five experimental rats in a within-subject-design experiment and also for all four experimental rats-but not at all for the four control rats-in a second, between-subjectdesign experiment. These results are interpreted within a framework that stresses three-term relations between $S, R$, and reinforcement that emphasizes the difference between earned and unearned reinforcement as two of those three terms.
\end{abstract}

In both classical and operant conditioning, the presentation of a substantial number of USs or reinforcers that are not preceded by a response or a punctate stimulus can result in substantial loss of conditioning (e.g., Rescorla, 1968; Rescorla \& Skucy, 1969). These poor conditioning situations are often described by the zero contingency or correlation between the two elements in conditioningstimulus and US or response and reinforcer (Rescorla, 1967).

In classical conditioning, some theories, such as the Rescorla-Wagner model (Rescorla \& Wagner, 1972), have emphasized the role that conditioning of the context plays in mediating such effects. According to this model, unsignaled USs condition the context or background cues, but when those USs are signaled by an entirely different CS, competition occurs between this new CS and the context. This view predicts that such added CSs before USs during the intertrial interval will make the zero contingency ineffective and that conditioning will now occur to the original CS. Most, but not all, experiments that have compared the effect of signaled versus unsignaled intertrial USs have supported this model. Durlach (1983) reports two such experiments and reviews many other such studies. In the classical conditioning literature, it can also be noted that there is a tangentially related effect of signaling intertrial USs upon the development or the maintenance of inhibition (Baker, 1977; Hammond, 1966, 1968).

The authors thank Philip Hineline for editorial assistance in the preparation of the manuscript. This research was supported in part by a grantin-aid from Temple University. Reprints may be obtained from the senior author at the Department of Psychology, Temple University, Philadelphia, PA 19122.
In operant conditioning, one can also arrange for a zero correlation or contingency between the elements of conditioning, in this case between response and reinforcement (RFT), and observe a loss of conditioning (see, e.g., Hammond, 1980). Since the unearned reinforcers occurring between the responses are somewhat analogous to the intertrial USs described above for the classical conditioning case, one can also examine the parallel question about the effect of signaling unearned reinforcers upon operant responding that is suppressed by a zero response-RFT contingency.

Although there is a parallel here between this manipulation for the two types of conditioning, there are also substantial differences between the two cases. A particularly relevant difference is that signaling unearned reinforcers in an operant paradigm will automatically involve the specification of three-term rather than two-term contingencies. This distinction, which was originally made by Skinner in 1938, has been elaborated by Catania (1971), Hammond (in press), and Jenkins (1977). Twoterm relations are those between stimuli and reinforcers (or CSs and USs) and responses and reinforcers. Threeterm relations involve stimuli, responses, and reinforcers. To signal an unearned reinforcer is to establish a relationship of the if-signal, and if-no-response, then RFT variety. Whether organisms respond to such three-term relations is an empirical question. As noted by Hammond (in press), there are eight possible such three-term relations (based on the combination of the presence and absence of the three terms). This experiment explores the effect of just one of these three-term relations (the ifstimulus, and if-no-response, then reinforcement relation), which we believe has not been investigated previously. 
In the research described below, rats' leverpressing was first established with constant probability schedules of RFT and then suppressed by the addition of equalprobability RFT for not responding (unearned RFT) following procedures used by Hammond (1980). This provided a baseline for evaluating the effects of signaling such unearned RFT.

\section{EXPERIMENT 1}

\section{Method}

Subjects. Five male albino rats, which weighed approximately $250 \mathrm{~g}$ each at the start of the experiment, had been obtained from Holtzman \& Co., Madison, Wisconsin, and had no previous experimental history, served as subjects. They were maintained on a 23-h-per-day water-deprivation schedule throughout the experiment.

Apparatus. The experimental spaces consisted of five Lehigh Valley Electronics (LVE) operant conditioning chambers, each equipped with one LVE lever to the right of the LVE water dipper and a $28-\mathrm{V}$ dc light with frosted-glass mounted just above the lever. Events in these chambers were scheduled by electromechanical equipment that included a paper-tape reader, all located in the next room. Responses were recorded on mechanical counters and monitored continuously on a 20-channel Esterline-Angus event recorder.

Procedure. After 2 weeks of handling and deprivation, the rats were given a single 1 -h session of magazine training in which they received $1000.03-\mathrm{cc}$ water reinforcers delivered at random times during the session. During the next 1-h session, the rats were exposed to continuous reinforcement training where every leverpress resulted in dipper operation. From the third daily session on, session length was $30 \mathrm{~min}$ and the procedure always consisted of some variation of the probabilistic schedule of RFT we have reported previously (Hammond, 1980).

It should be noted that schedule procedures like the one used here were originally described by Schoenfeld (Schoenfeld, Cummings, \& Hearst, 1956) and were called random-interval or random-ratio schedules. However, these terms have often been used to label schedules that are at variance with Schoenfeld's original criteria (e.g., Millenson, 1963) and not really constant probability schedules of reinforcement delivery, since they contain unlimited holds of reinforcement. So we will describe what was used here as a probabilistic schedule, since it maintains a constant probability of RFT given a leverpress in any second throughout the entire session. With this procedure, there is no "setting-up" and "holding"' of RFT as is typical of conventional interval schedules-a practice that drastically affects the probability distribution of RFT.

In the specifics of the present procedure, the programming equipment divided the session into 1-sec segments. At the end of each segment, water delivery was probabilistically allocated. If one or more responses had been made in that second, one channel of paper tape was used, but if no response occurred in that second, a second channel was used to determine RFT. With constant, but independent, probabilities of holes in the two channels of the tape, probability of RFT for responding (R) and for not responding (not R) was held constant throughout the entire session. The two parameters of this schedule were the RFT probabilities for $R$ and not $R$, and each schedule was described by two percentages of RFT per second, the first for $\mathbf{R}$ and the second for not $\mathbf{R}$ (e.g., $5 \%-0 \%$ ).

During some phases of the experiment, we added a not-R water delivery (our "unearned" reinforcer) where one had not initially occurred (e.g., from 5\%-0\% to $5 \%-5 \%$ ), and then, in another change of procedure, we added a signal before each of these unearned reinforcers. The experiment consisted of variations in these features; the sequence of the procedure changes is shown in Table 1 . Each rat received identical treatment.
Table 1

Summary of Experimental Conditions in Experiment 1

\begin{tabular}{clc}
\hline $\begin{array}{c}\text { Phase of } \\
\text { Experiment }\end{array}$ & \multicolumn{1}{c}{$\begin{array}{c}\text { Condition } \\
\text { (R-not R) }\end{array}$} & $\begin{array}{c}\text { Number of } \\
\text { Sessions }\end{array}$ \\
\hline 1 & Magazine training & 1 \\
2 & Continuous reinforcement & 1 \\
3 & $20 \%-0 \%$ & 1 \\
4 & $12 \%-0 \%$ & 4 \\
5 & $5 \%-0 \%$ & 7 \\
6 & $5 \%-5 \%$ & 22 \\
7 & $5 \%-5 \%$ with signal & 30 \\
8 & $5 \%-5 \%$ & 30 \\
9 & $5 \%-0 \%$ & 25 \\
10 & $5 \%-5 \%$ & 30 \\
11 & $5 \%-5 \%$ with signal & 33 \\
\hline
\end{tabular}

To summarize this table, rats were initially shifted through decreasing probabilities, but with high positive contingency, until they had been on a 0.05 probability of RFT for $R$ and a 0.0 probability of RFT for not-R (5\%-0\%) schedule without any signals (Phase 5). Then, while the probability of RFT for R was held constant, they were shifted to a zero-contingency schedule, $5 \%-5 \%$, but still with no signal (Phase 6). In Phase 7, a 5-sec signal was added to each programmed not-R RFT while exactly the same $5 \%$ $5 \%$ schedule as in Phase 6 was maintained. If the rat responded in the last second of that signal, this prevented the occurrence of the progammed "unearned" RFT (as would be the case on an unsignaled $5 \%-5 \%$ ).

At any time during the signal, however, an $\mathrm{R}$ could produce "earned" RFT ( $5 \%$ of the time). In actual occurrence, then, not all flashing lights terminated in RFT, but all "unearned" RFTs that occurred were signaled. During a typical 30 -min session, an average of 90 signals occurred and therefore one-fourth of the session was occupied by signals. In Phase 8 , the signals were discontinued and all other procedural details remained the same as in Phase 6 (e.g., an attempt was made to recapture the zero-contingency baseline). In Phases 9 through 11, the experimental sequence of positive contingency, zero contingency, and zero contingency plus signal was repeated with the same parameters as before.

\section{Results and Discussion}

Individual response rates (per each 30-min session) for all five rats for Phases 5 through 11 appear in Figures 1 and 2 . Each phase is represented as a column of panels and each row represents a separate rat. In agreement with previous work on shifts in contingency with these probabilistic schedules (Hammond, 1980), all five rats showed substantial declines in responding when shifted from the positive contingency of Phase 5 to the zero contingency of Phase 6, and in four of five cases that decline was almost a full scale decline. The transition to Phase 7 is the novel manipulation of the present study. When the signal was added to the suppressed responding produced by the degraded contingency, all five rats showed at least a twofold increase in response rate.

A percentage of the Phase 5 baseline score was calculated for each rat, based on the mean response rate during the last 3 sessions of Phase 5 and the mean response rate during the last 10 sessions of either Phase 6 or Phase 7 (to generate two different scores for each rat). The mean percent baseline score for all five rats toward the end of the unsignaled zero-contingency phase (6) was $21.8 \%$, 


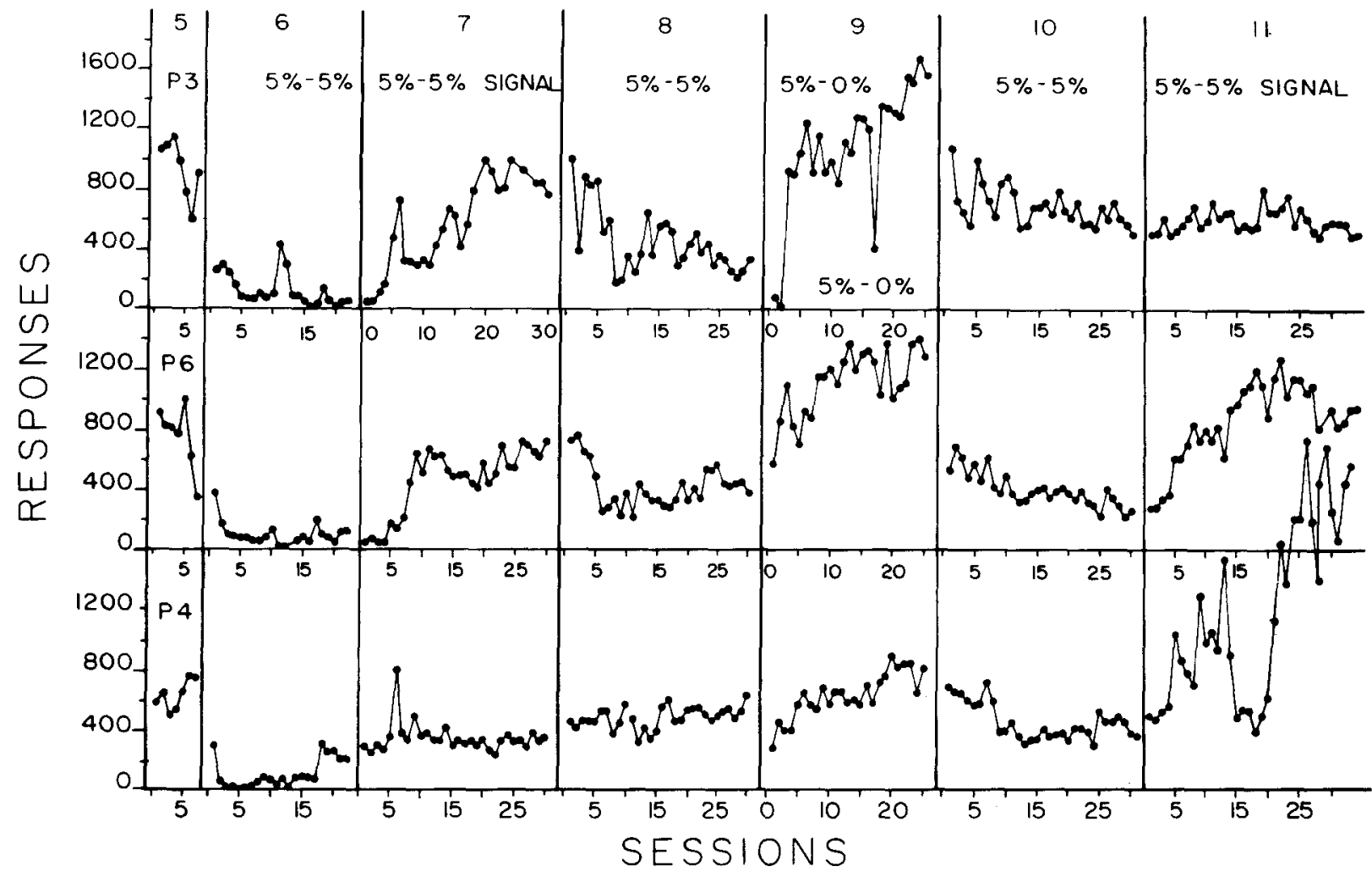

Figure 1. Total responses for each 30-min session for rats P3, P6, and P4 during Phases 5 through 11 of Experiment 1 . Each row represents one rat; each panel represents one phase of the experiment.

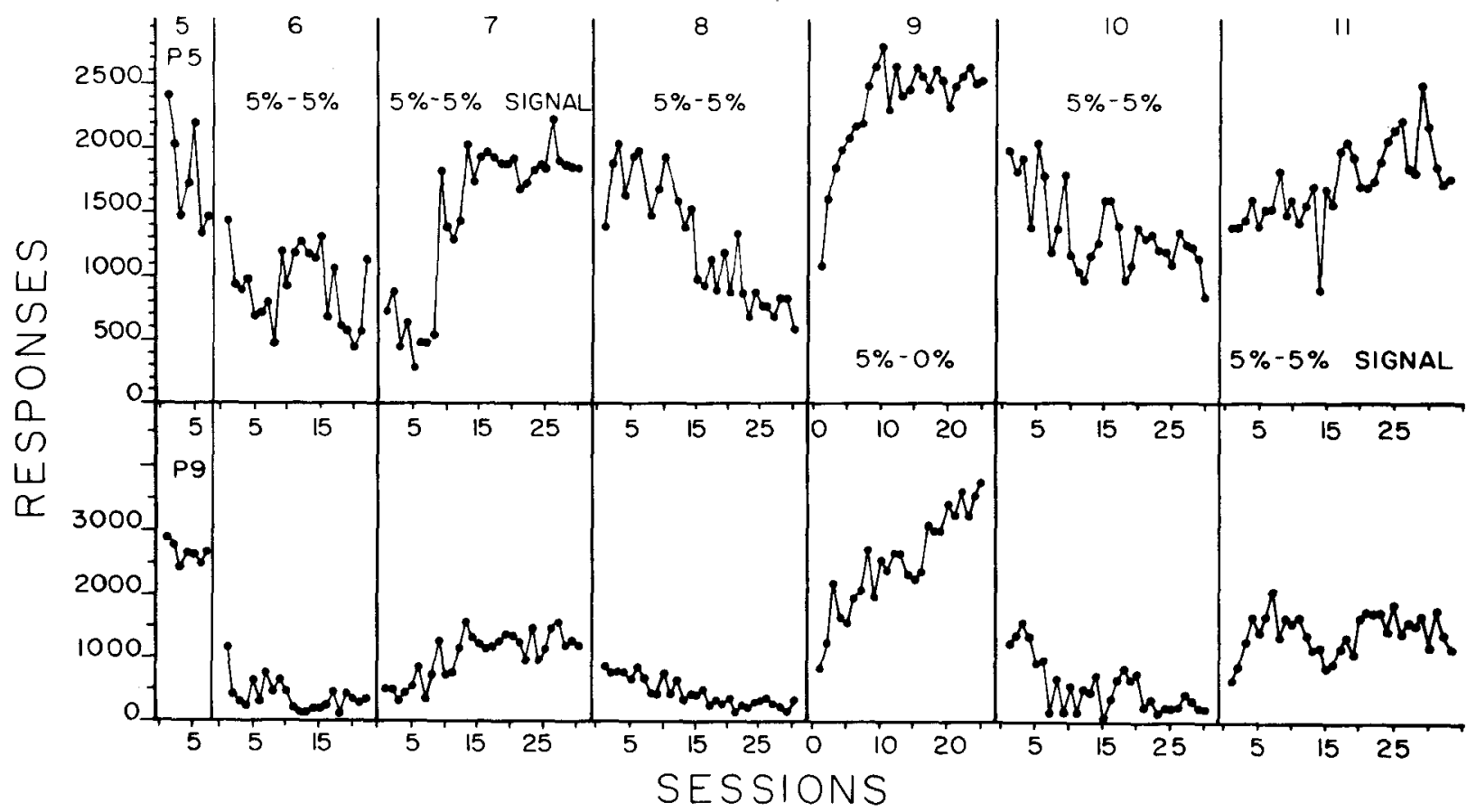

Figure 2. Total responses for each 30-min session for rats P5 and P9 during Phases 5 through 11 of Experiment 1. 
and toward the end of the signaled zero-contingency phase (7), 74.6\%. Thus, if we use the Phase 5 positive contingency as a reference condition, the signal manipulation produced a shift in performance of one-half of the scale (with reference to Phase 5), and this one-half scale manipulation resulted in a threefold increase in the zerocontingency response rate.

Phase 8 involved an attempt to recapture the rat's performance under an unsignaled zero contingency, and in this regard the manipulation was not successful. Although four of the five rats showed a decline in response rate from Phase 7 to Phase 8 , only one rat (P9) declined to the level seen previously under the zero contingency (Phase 6).

In the next phase (9), the rats were returned to the positive contingency of Phase 5 (5\%-0\%) to see if that baseline could be recovered. As can be seen from the Phase 9 column of Figures 1 and 2, the manipulation was successful in producing increased response rates; however, four of the five rats responded at higher levels in Phase 9 than in Phase 5, which had involved the identical procedure.

In Phase 10, the rats were returned to the zero contingency a third time, preparatory to replicating the major manipulation of the study-signaled RFT of the noresponse category, which was done in Phase 11. The zero contingency produced sharp declines relative to the preceding phase (9) for three animals. For two rats, P3 and P5, however, this zero contingency baseline was not completely replicated; the other three rats declined to the same level as in the previous zero contingency of Phase 8 . Upon reintroduction of the signal manipulation, four of the five rats showed once again a substantial increase in response rate to levels surpassing the absolute level shown when the signal was introduced before (Phase 7). One rat, P3, did not show any effect of the added signal in the replication, although he had shown an effect originally.

The effect of the signal does not appear to be a simple unconditioned effect of the light. If that were the case, one would expect an immediate increase in responding once the signals were introduced. Instead, what occurred was a very gradual increase in responding over many sessions of exposure to the signal condition.

In summary, the major focus of this experiment was upon the effect of signaling unearned RFT during zerocontingency suppression of an operant. This manipulation was introduced twice with five rats for a total of 10 times. In 9 of these 10 instances, the zero-contingency suppression was attenuated to a marked degree. We will turn to a discussion of why this was occurring after describing our second experiment, in which we wished to examine several concerns that we had about the first experiment.

\section{EXPERIMENT 2}

In the experimental analysis of behavior, control over extraneous influences upon the measured behavior is often accomplished by repeating a before-after experimental manipulation. Such repeated demonstrations are all the more convincing if removal of the major manipulation results in a return to performance closely similar to that seen before the manipulation was introduced. In Experiment 1 , the absolute level of responding under the zerocontingency condition was often quite different from one replication to the next, and this detracted to some extent from the demonstration of the effect of the signals. The fact that the level of responding was greater during exposure to the second zero contingency than during exposure to the first is opposite to what had been observed in earlier work (see Figure 1; Hammond, 1980). This finding raised the related question of what would have happened to the operant performance if the rats had been maintained on the zero contingency without the addition of any signals. Would this length of exposure to the zero contingency by itself lead to an increase in operant responding?

In an attempt to remove the confounding discussed above, the signal manipulation in Experiment 2 was examined between subjects rather than within subjects, since a between-subjects comparison would remove concern about baseline drift in general. At the same time, we wished to closely examine responding during the signal as well as in its absence, since casual observation of the response counters in Experiment 1 had suggested that considerable suppression was taking place during the signal.

Thus, in Experiment 2, one group of rats was exposed to the positive contingency, zero, and then zero plus signal manipulations, as in the first part of Experiment 1. This time, however, a control group of rats had identical treatment but without the addition of any signals. Also, toward the end of the experiment, we measured suppression to the signal in the experimental group.

\section{Method}

Subjects. Eight male albino rats that had been obtained from Holtzman \& Co., Madison, Wisconsin, weighed approximately $250 \mathrm{~g}$, and had no previous experimental history served as subjects.

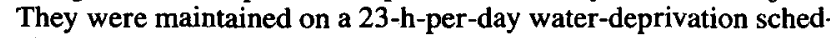
ule throughout this experiment.

Apparatus. The apparatus was identical to that of Experiment 1, except that eight chambers were used instead of five.

Procedure. These eight rats received the same preliminary handling, deprivation, and initial training as the subjects in Experiment 1 up through Phase 3. All treatment phases for the rats in Experiment 2 are shown in Table 2 . The differences between the

Table 2

Summary of Experimental Conditions in Experiment 2

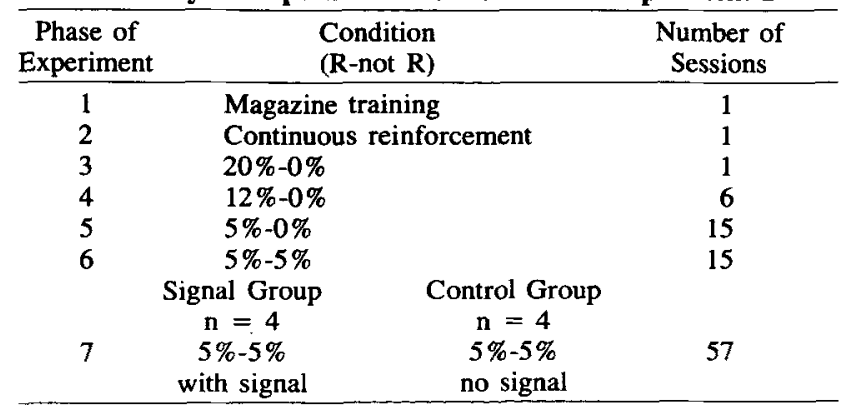




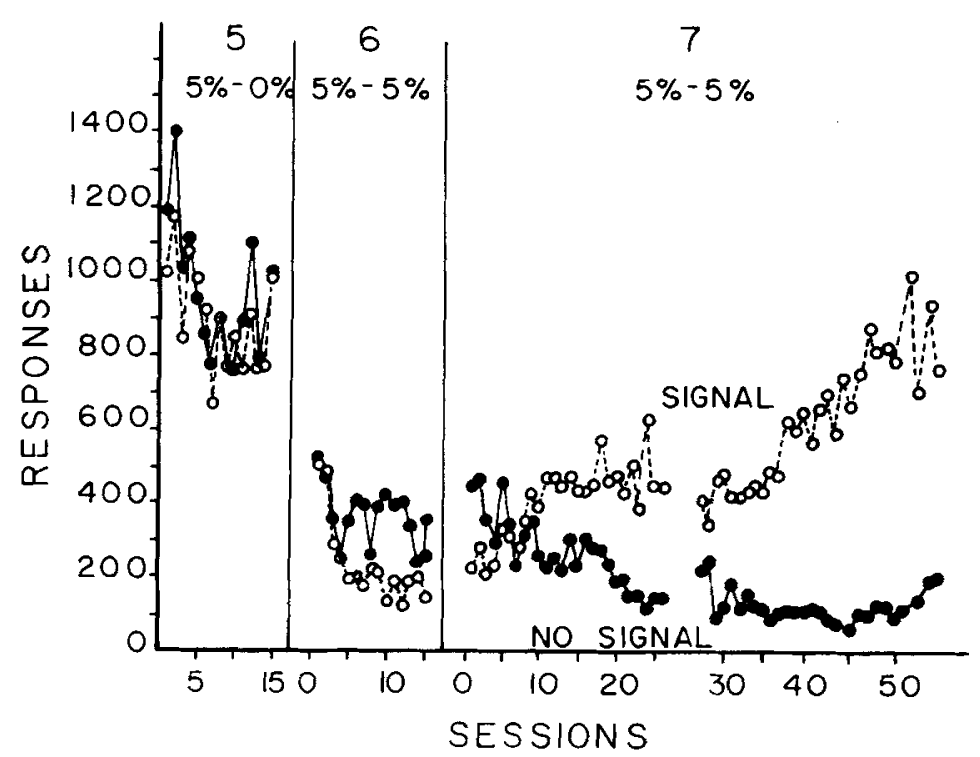

Figure 3. Mean responses for each 30-min session of Experiment 2 for the four rats in the signal group and the four rats in the no-signal group plotted across the sessions of Phases 5 through 7. The gap in the curves during Phase 7 represents the long "vacation" described in the text.

two experiments are revealed by comparing Table 1 with Table 2 . The most important differences occurred in Phase 7: (1) After Phase 6, the first unsignaled zero contingency, the eight rats were divided into two groups of four rats each. One group was maintained on the zero contingency without a signal, whereas the other, experimental, group continued on the same zero contingency, 5\%$5 \%$ schedule, except that they were given a signal before each unearned reinforcement in exactly the same manner as signals were delivered in Experiment 1 , (2) No replications were performed, and there were no further phases. (3) There was a prolonged 5-week "vacation" between the 26th and 27th session of Phase 7. Otherwise, the rats were exposed to a 5-day-a-week running schedule, as before.

\section{Results and Discussion}

Figure 3 shows the mean response rates, again per 30min session, for the signal and control groups of rats for Phase 5 (positive contingency, 5\%-0\%), Phase 6 (zero contingency, no signal, 5\%-5\%), and Phase 7 (zero contingency, signal for one group only, $5 \%-5 \%$ for both). The four new animals in the signal group performed in the same manner as did the five rats in Experiment 1. They responded substantially on the positive contingency (Phase 5), declined dramatically when the zero contingency was introduced (Phase 6), but resumed responding gradually when each "unearned" RFT was preceded by a signal (Phase 7), until finally the mean rate of responding was approximately as high at the end of Phase 7 as it had been at the end of Phase 5. Thus, the findings of the signal group replicated those for the rats in Experiment 1. The control group showed the same mean amount of responding on the positive contingency (Phase 5), declined during the zero contingency (Phase 6), though not quite as much as did the signal group, and then, in Phase 7, while still exposed to the same zero contin- gency as in Phase 6, continued its gradual decline in responding. Thus, a major goal of Experiment 2 was accomplished-the demonstration that prolonged exposure to a zero contingency does not lead to release from the initial suppression or a return to baseline responding.

Critical aspects of individual performance for rats in both groups are shown by the histogram in Figure 4 .

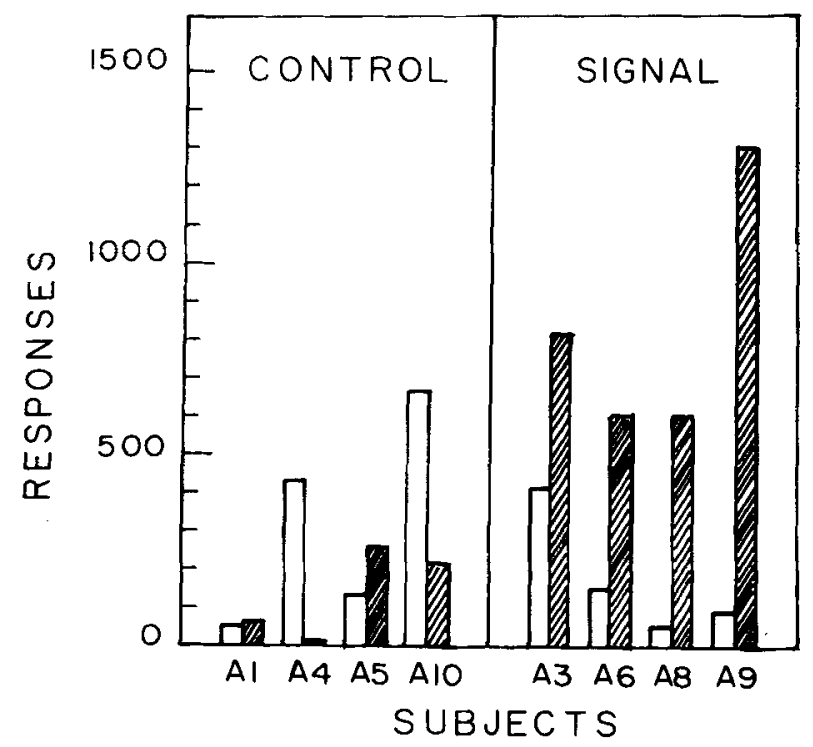

Figure 4. A histogram for each individual rat in the control group (left panel) and each rat of the experimental group (right panel) of Experiment 2 showing the mean session response totals based on the $\mathbf{1 0}$ sessions before signal introduction (clear bars) and the last 10 sessions of the signal procedure (shaded bars). 
Mean session response totals for the last 10 sessions before the signal was introduced (Sessions 5-15 of Phase 6 are compared with mean session response totals for the last 10 sessions at the end of the experiment (Sessions 4757 of Phase 7) for each of the eight rats. As can be seen, all four of the signal rats showed a greater increase in responding from the before measure to the after measure than any of the four control rats, and this effect was significant (Mann-Whitney $U$ test, $U=0, n_{1}=4, n_{2}=$ $4, p=.028$, for a two-tailed test).

Once again, there is the issue of unconditioned light effects. Examination of Figure 3 shows that the attenuation of the suppressive effects of the zero contingency by the addition of the light signals was an extremely gradual phenomenon, and the bulk of the effect did not appear until after 40 sessions of the signal procedure, during which each rat had received 3,600 light exposures. This is not what we would expect from a simple unconditioned stimulus effect.

Finally, there is the matter of responding during the 5sec signals, which collectively amounted to $25 \%$ of each session for the signal rats in Phase 7. In accordance with the casual observations of the rats in Experiment 1, each of the four signal rats showed substantial suppression of responding during the lights. Such responding was sampled during two blocks of sessions, from Session 38 to Session 41 and then the last five sessions of Phase 7, Sessions 53-57. Responses during all lights were recorded during each of these blocks of sampled sessions. Also, a comparable length of time in the absence of the signals was sampled from each of these sessions to provide a baseline. These no-light recording periods were selected such that no RFTs, earned or unearned, were programmed during these brief baseline periods. In this way, a measure of responding unconfounded by drinking as obtained for no-light and light conditions, such that a typical suppression ratio (light/light + no light) could be calculated for the effects of the light on leverpressing.

During the first sample block of sessions, the mean suppression ratio was .18 (range .05-.46), whereas in the later sample block of sessions, the mean was .09 (range .01.26). Obviously, there was substantial suppression to the light signal and the overall effect of signaling unearned reinforcers as seen in Figure 3 must be evaluated in view of the fact that practically no responses were made during $25 \%$ of each sesion. Since total responses per session are plotted in Figure 3, it may be argued that those responses are occurring almost entirely in the absence of the signal.

\section{GENERAL DISCUSSION}

The effect of signaling unearned RFT was quite striking in our experimental situation. When the signal was not on, the rats went back to work. For most of the rats, the suppressive effects of unearned RFT, and therefore the zero contingency, were completely eliminated by these brief signals.
As noted in the introduction, the signaling of intertrial USs in classical conditioning can remove the debilitating effects these "free" USs have upon the learning or display of the CR (e.g., Durlach, 1983). Our findings extend this phenomenon to the suppressive effects of unearned RFT upon operant responding. At least one very important distinction between the two cases (classical and operant) is based on the number of terms that are involved in the relationship. That is, although one can describe classical conditioning as based on the relationship between CS and US and operant conditioning as based on the relationship between R and RFT (each a two-term relation), in the operant case one can also specify three-term relations involving $S, R$, and RFT. The traditional three-term relations of operant discrimination stressed the if-S-andif-R-then RFT relation (conditions for a discriminative stimulus) and the if no $S$ (or a different $S$ ) and the if-Rthen-no-RFT relation (conditions for an S-delta). Hammond (in press) has discussed six other three-term relations, four of which deal with predictions about the occurrence or nonoccurrence of unearned RFT. Only one of these relations was examined in this study-the if-Sand-if-no-R-then-RFT relation. In this study, we have shown that one of these relations can exert powerful discriminative control over behavior-perhaps organisms will be sensitive to the other three-term relations concerned with unearned RFT as well. We hope to explore that in future research.

The procedures used in this study were originally designed (Hammond, 1980) to examine a particular form of probabilistic contingency theory which has often been called the delta-p, or probability difference, formulation (Hammond \& Paynter, 1983). According to this formulation, when the probability of RFT given a response is equal to the probability given no response, then no conditioning should occur, but as the probability of RFT given a response becomes larger than the probability given no response, conditioning should become stronger. This formulation has been quite useful for the restricted cases that were explored in the present study. For example, under the original "zero"' contingency before the signal was introduced, the probability of RFT given both $\mathrm{R}$ and not $R$ was .05. After the brief signals were introduced just preceding the unearned RFTs, these probability differences could then be recalculated for the signal and nonsignal periods. During the signal, the probability of RFT per second given no $R$ was .20, where the RFT probability given no $R$ was .00 in the absence of the stimulus. Since the consequences of responding were always the same (RFT probability given $R=0.05$ ) both in the presence and in the absence of the signal, a positive contingency existed in the absence of the signal and a negative contingency existed in the presence of the signal. Thus, the delta-p formula would predict substantial responding in the absence of the signal and suppression in the presence of the signal, and that of course is what we found. As is the case with all probabilistic contingency theories, however, the delta-p formulation stumbles in less 
friendly environments, as is discusssed in Hammond and Paynter (1983).

\section{REFERENCES}

BAKER, A. G. (1977). Conditioned inhibition arising from a betweensessions negative correlation. Journal of Experimental Psychology: Animal Behavior Processes, 3, 144-155.

CATANIA, A. C. (1971). Elicitation, reinforcement, and stimulus control. In R. Glaser (Ed.), The nature of reinforcement (pp. 196-220). New York: Academic Press.

DuRLACH, P. J. (1983). The effect of signaling intertrial USs in autoshaping. Journal of Experimental Psychology: Animal Behavior Processes, 9, 374-389.

HAMmOND, L. J. (1966). Increased responding to CS - in differential CER. Psychonomic Science, 5, 337-228.

HAMmOND, L. J. (1968). Retardation of fear acquisition when the CS has previously been inhibitory. Journal of Comparative and Physiological Psychology, 66, 756-759.

HAMMOND, L. J. (1980). The effect of contingency upon the appetitive conditioning of free-operant behavior. Journal of the Experimental Analysis of Behavior, 34, 297-304.

HAMmOND, L. J. (in press). An empirical legacy of two-process theory: Two term versus three term relations. In F. R. Brush \& J. B. Overmier (Eds.), Affect, conditioning and cognition: Essays on the determinants of behavior. Hillsdale, NJ: Erlbaum.

Hammond, L. J., \& PAYNTER, W. E., JR. (1983). Probabilistic contin- gency theories of animal conditioning: A critical analysis. Learning and Motivation, 14, 527-550.

JENKINS, H. M. (1977). Sensitivity of different response systems to stimulus-reinforcer and response-reinforcer relations. In $\mathbf{H}$. Davis \& H. M. B. Hurwitz (Eds.), Operant-Pavlovian interactions (pp. 4762). Hillsdale, NJ: Erlbaum.

Millenson, J. R. (1963). Random interval schedules of reinforcement. Journal of the Experimental Analysis of Behavior, 6, 437-443.

RESCORLA, R. A. (1967). Pavlovian conditioning and its proper control procedures. Psychological Review, 74, 71-74.

Rescorla, R. A. (1968). Probability of shock in the presence and absence of CS in fear conditioning. Journal of Comparative and Physiological Psychology, 66, 1-5.

RESCORLA, R. A., \& SKUCY, J. C. (1969). Effect of response-independent reinforers during extinction. Journal of Comparative and Physiological Psychology, 72, 381-389.

Rescorla, R. A., \& Wagner, A. R. (1972). A theory of Pavlovian conditioning: Variations in the effectiveness of reinforcement and nonreinforcement. In A. H. Black \& W. F. Prokasy (Eds.), Classical conditioning II: Current research and theory (pp. 644-99). New York: Appleton-Century-Crofts.

Schoenfeld, W. N., Cummings, W. W., Hearst, E. (1956). On the classification of reinforcement schedules. Proceedings of the $\mathrm{Na}$ tional Academy of Science, 42, 563-570.

SKINNER, B. F. (1938). The behavior of organisms. New York: Appleton-Century-Crofts.

(Manuscript received August 29, 1983; revision accepted for publication July 17, 1984.) 